\title{
UBICUIDAD DUAL: BASE PARA LA EFECTIVIDAD DEL VRCINEMA COMO HERRAMIENTA PROSOCIAL. ANÁLISIS DE HUNGER IN L.A. Y AFTER SOLITARY
}

\author{
Dr. Francisco-Julián Martínez-Cano \\ Universidad Miguel Hernández, Elche, España \\ francisco.martinezc@umh.es \\ ORCID iD: https://orcid.org/0000-0001-8551-3144 \\ Dra. Begoña Ivars-Nicolás \\ Universidad Miguel Hernández, Elche, España \\ bivars@umh.es \\ ORCID iD: https://orcid.org/0000-0003-1127-0607 \\ Dra. Alba-María Martínez-Sala \\ Universidad de Alicante, Alicante, España \\ albamaria.martinez@ua.es \\ ORCID iD: https://orcid.org/0000-0002-6852-6258
}

Recibido el 30 de septiembre de 2019

Aceptado el 31 de enero de 2020

\section{Resumen}

El medio audiovisual ha servido a lo largo de su historia como transmisor de valores. Desde el cine militante al videoactivismo, las producciones audiovisuales han sido agentes generadores de conciencia en el espectador que, con la evolución del ecosistema mediático y la irrupción de la realidad virtual (RV), ofrecen nuevas posibilidades. Entre ellas, la ubicuidad dual, clave en la generación de empatía en la audiencia, ya que es capaz de transportarla a otras realidades. Por tanto, ¿qué impacto puede tener la realidad virtual en el ámbito del activismo audiovisual contemporáneo? Para profundizar sobre esta cuestión, se ha llevado a cabo una revisión bibliográfica de los referentes, así como de las últimas investigaciones que abordan este tema. Una vez completada esta primera fase, diseñamos un modelo de análisis de películas de realidad virtual, que actualiza el triángulo inmersivo (Cortés-Selva, 2015), a partir de las variables que establecen Archer y Finger (2018) y Domínguez-Martín (2015), con el que se han analizado dos producciones de Emblematic Group, una de las principales productoras del ámbito de la no ficción con RV. Finalmente, en los resultados se exponen algunas de las características comunes, identificadas como necesarias, para producir un impacto emocional en la audiencia, indispensable para su eficacia como herramientas de transformación social.

Palabras clave: cine de realidad virtual, videoactivismo, ubicuidad dual, narración inmersiva, presencia, entornos virtuales. 


\title{
DUAL UBIQUITY: BASIS FOR THE EFFECTIVENESS OF THE VRCINEMA AS A PROSOCIAL TOOL. ANALYSIS OF HUNGER IN L.A. AND AFTER SOLITARY
}

\begin{abstract}
The audiovisual medium has served as a transmitter of values throughout its history. From militant cinema to videoactivism, audiovisual productions have been agents that generate awareness in the viewer, and currently offer new possibilities through the evolution of the media ecosystem and the emergence of virtual reality (VR). Among them, dual ubiquity, key in the generation of empathy in the viewer, since it is capable of transporting the audience to other realities. Therefore, what impact can virtual reality have on the field of contemporary audiovisual activism? To deepen this issue, a bibliographic review of the references has been carried out, as well as on recent research that addresses this issue. Once this first phase is completed, we design a virtual reality film analysis model that updates the immersive triangle (Cortés-Selva, 2015), based on the variables established by Archer and Finger (2018) and Domínguez-Martín (2015), with which two productions of Emblematic Group, one of the main producers of the field of non-fiction with RV, have been analyzed. Finally, the results show some of the common features identified as necessary to produce an emotional impact on the audience, essential for their effectiveness as tools of social transformation.
\end{abstract}

Keywords: virtual reality cinema, videoactivism, dual ubiquity, immersive narration, presence, virtual environments. 


\section{Introducción}

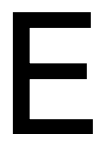

I cine, desde desde su nacimiento como medio de comunicación de masas, ha sido utilizado como herramienta política y con fines de adoctrinamiento en valores e ideales. Ha sido transmisor de convenciones culturales e instrumento de propaganda por parte de diferentes regímenes políticos y gobiernos. Desde las producciones cinematográficas comerciales de Hollywood, ya se atisba un discurso que transporta de manera indirecta una serie de connotaciones políticas, con el fin de colonizar culturalmente otras naciones desde el consumo de sus producciones, "Todo cine al ser vehículo de ideas y modelos culturales, e instrumento de comunicación y proyección social, es en primer término un hecho ideológico, y en consecuencia también un hecho político" (Getino y Solanas, 1973, citado en Galán, 2012a: 1123).

\subsection{Activismo audiovisual en el siglo $X X$ y $X X \mid$ : del cine militante al cine de realidad virtual}

Ya en la década de los 60 y 70, y al margen de la industria cinematográfica, surge en el contexto internacional el denominado cine militante, dando lugar a una práctica fílmica basada en la producción audiovisual como herramienta de transformación social. "El cine militante rechaza la visión de las películas como un objeto de consumo que se presenta como puro divertimento, una fórmula ejemplarizada en el cine de Hollywood" (García-Merás, 2007: 17). Su definición por Fernando Solanas y Octavio Getino (1973), indica diferentes posibilidades dentro de sus objetivos:

Cine militante es aquel cine que se asume integralmente como instrumento, complemento o apoyatura de una determinada política y de las organizaciones que la lleven a cabo, al margen de la diversidad de objetivos que procure: contrainformar, desarrollar niveles de conciencia, agitar, formar cuadros, etcétera [...] (Solanas y Getino, citado en Mestman, 2001: 2)

En las últimas décadas del siglo XX, con la aparición de las cámaras de video compactas y el abaratamiento de los costes, se socializa el acceso a las tecnologías audiovisuales dando lugar al videoactivismo, movimiento heredero del cine militante, que se focaliza en la producción de metraje, con urgencia de contar aquello que acontece, y que en muchos casos los medios tradicionales no incluían en su discurso. Debido a esta premura, las características fundamentales de la práctica videoactivista es la producción rápida del contenido, cámara al hombro, sin escrúpulos estéticos ni planificación premeditada, el uso de planos generales y la participación del realizador dentro de la acción, bien, a través de la voz en off o bien, apareciendo directamente en cuadro, incluso dirigiéndose a los actores de aquello que registra, del acontecimiento en sí, de un modo espontáneo, cuya edición final también se basa en la rapidez, donde el corte es el método de montaje principal y se obvian los procesos y efectos más complejos de posproducción. El plano sonoro se compone del registro realizado durante la grabación, sin añadidos 
ni bandas sonoras, con el fin de sumergir al espectador e implicarlo de un modo más directo con el conflicto presentado.

A las prácticas videoactivistas, en un sentido estético y narrativo, se las puede considerar herederas de los informativos televisivos donde las dinámicas del directo condicionan los discursos audiovisuales. En una sociedad completamente mediatizada, la noticia se convierte en la auténtica protagonista. El directo en las televisiones se convierte en un valor en sí mismo, modificando la relación del medio televisivo con la realidad (Galán, 2012a: 1128).

Estas corrientes resultan en el activismo audiovisual actual, heredero del cine militante y del videoactivismo, de las décadas de los 80 y 90, que amplifica la difusión de sus obras y, por tanto, su impacto a través del desarrollo y evolución de las tecnologías de la información e Internet. Una definición posible para este paradigma de acción social, válida para todos sus momentos históricos, es la que aporta Luis Herrero:

Genéricamente se utiliza el término de videoactivismo para significar todas aquellas acciones de contestación de amplio espectro político, social y artístico que utilizan el vídeo como herramienta y encuentran en las nuevas tecnologías su medio natural de creación y difusión (Luis Herrero, 2008, citado en Galán, 2012b: 7).

Además, esta proliferación del audiovisual como herramienta de transformación social, en el contexto actual, inmersos en continuo contacto con los medios, a través de la omnipresencia de canales de información en los flujos de Internet, las redes sociales, las plataformas de video bajo demanda, y el uso de dispositivos móviles que facilitan el acceso constante a la información, dan lugar al concepto de ubicuidad, el cual puede apuntarse como una de las últimas estrategias de generación de impacto de estas prácticas activistas audiovisuales. La experiencia vital contemporánea se caracteriza de una mezcla de lo real y lo virtual, a través de los procesos de ubicuidad. Nos llegan inputs procedentes de otros lugares, con contenidos en formato audiovisual que nos acercan a otras realidades ajenas a la nuestra. En definitiva, vivimos inmersos en la sociedad ubicua, término acuñado por Kunio Makamura en el Ceatec 2004:

El término "sociedad de la ubicuidad", afirma Nakamura, designa a una sociedad en la que cualquier persona puede disfrutar, en cualquier momento y en cualquier lugar, de una amplia gama de servicios de información y a través de diversos dispositivos terminales y redes de banda ancha. La importancia de las comunicaciones digitales móviles se encuentra implícita en el lema de la sociedad de la ubicuidad: "anyone, anywhere, anytime" (Islas-Carmona, 2008: 33).

Los individuos de la sociedad ubicua son, por tanto, personas ubicuas, cuya definición encontramos en el texto de José Antonio Gabelas Barroso, Carmen Marta Lazo y Elisa Hergueta Covacho, titulado Comunicación, ubicuidad y aprendizajes, presentado en el IV Congreso Internacional Latino de Comunicación Social: 
Hablamos de alguien cuya meta es tener acceso ilimitado a la mayor cantidad de información posible de manera descentrada. Si transferimos esta información al término ubicuidad, vemos que podemos definirla como la capacidad de estar presente y en movimiento de manera ilimitada. Conectándonos a través de la tecnología a fuentes de información y de conocimiento formamos redes, no sólo con personas, sino también con esas fuentes que median nuestra comunicación y nuestra necesidad de información y nos ayudan a acceder y a ampliar nuestras habilidades cognitivas $y$, a gestionar de esta manera, nuestra identidad y nuestro conocimiento (Barroso, Lazo y Covacho, 2012: 8).

La irrupción de la realidad virtual (RV) como herramienta de generación de contenidos audiovisuales, y el comienzo de su estandarización como medio de consumo de discursos cinemáticos, en las primeras décadas del siglo XXI, da lugar a la aparición de las primeras prácticas cinematográficas con este medio, así como de una nueva corriente de activismo audiovisual, que aprovecha el poder inmersivo de estos discursos y su factor de presencia, para elaborar obras en las que el espectador es introducido en el lugar del relato. Mateo Pasquinelli hace referencia al poder de los dispositivos y medios audiovisuales y su potencial transformador: "En las manos de un videoactivista, una telecámara puede funcionar como disuasor contra la violencia de la policía, un sistema de monitoreo por vídeo puede influenciar la agenda política, un proyector de vídeo puede generar conciencia colectiva" (Mateo Pasquinelli, 2002, citado en Galán, 2012b: 8). Por tanto, ¿qué impacto puede tener la realidad virtual en el ámbito del activismo audiovisual contemporáneo?

\subsection{Ilusión de lugar, plausibilidad y corporalidad virtual. Elementos clave de la ubicuidad dual}

En relación directa con el activismo audiovisual y sus movimientos, surge un nuevo paradigma narrativo, que impulsan la reflexión social y política de nuestro entorno, con el fin de generar conciencia en el espectador y transformar la sociedad actual, aprovechando las características de la RV y del videojuego, su uso y aplicación para la creación de contenidos inmersivos 3D e interactivos. Una de las primeras autoras en este ámbito, Nonny de la Peña, ha confeccionado un cuerpo de obra virtual partiendo de la práctica periodística, dando lugar al denominado periodismo inmersivo. Primero con el grupo Immersive Journalism, con el apoyo de la USC Annenberg School for Communication, y actualmente con Emblematic Group, cuya carta de presentación en su web (https://emblematicgroup.com) ya indica las principales características e innovaciones con las que su equipo multidisciplinar trabaja:

Fundada en 2007 por Nonny de la Peña, conocida como la "madrina de la realidad virtual", el equipo de galardonados periodistas, fanáticos de los medios de comunicación, cineastas y desarrolladores lideran la industria en la creación y puesta en escena de entornos de realidad virtual a escala de habitación, que sitúan al usuario en la escena de la historia, lo que les permite moverse a través de la acción (Emblematic Group, 2019). 
El mito del "cine total" de Bazin (1966: 17-22) comienza a convertirse en realidad. La ilusión de lugar, la plausibilidad (o verosimilitud) y la corporalidad virtual (o apropiación del cuerpo virtual) (Peña et al, 2010: 294), son los principales conceptos que funcionan como pilares para la creación de experiencias inmersivas, capaces de sumergir al usuario como sujeto activo de la narración, accediendo al contenido audiovisual y a los sentimientos y emociones que de este se desprenden. "Un sistema de realidad virtual capaz de ofrecer estos tres conceptos, proporciona los medios para transformar no solo la sensación espacial y de realidad de la audiencia sino también a los propios espectadores" (Peña et al, 2010: 295).

La simulación es la estrategia principal de la construcción del relato audiovisual en formato virtual, desde la representación de un acontecimiento a partir de audio y video real, reconstruyendo los hechos desde una perspectiva periodística y transitando el género documental, con el fin de aumentar la empatía del público y la comprensión de una realidad presente a través de la ubicuidad. Partiendo del objetivo de crear experiencias audiovisuales inmersivas, se ha trabajado con diferentes medios y soportes para generar sensación de presencia, lo que supone colocar a la audiencia dentro de la imagen:

La imagen inmersiva periodística utiliza actualmente distintas técnicas, como el video y fotografía en 360 grados, panorámicas, reconstrucciones creadas con imagen cien por ciento digital (se están adaptando entornos como Unity fundamentalmente utilizadas en el ámbito de la ficción digital) o producciones para Oculus Rift. De todos ellos, la fotografía en 360 grados es el formato más veterano. Actualmente la integración del sonido ambiental en ellos ha intensificado la sensación de presencia, de "estar allí". El sonido constante, sutil y ambiental contribuye a crear una atmósfera, un clima (Domínguez-Martín, 2015: 418).

Estas experiencias inmersivas unen la práctica periodística y la cinematográfica a través del empleo de la RV como medio y soporte. Se trata, por tanto, de un cine de realidad virtual (VRCinema) que aborda el género de la no ficción a partir de los modos de construcción periodísticos, basados en registros documentales reales de los acontecimientos reconstruidos. Estas obras adquieren una función de transformación social desde el punto de vista de situar al espectador dentro de la acción del discurso, alcanzado altos niveles de empatía y concienciación respecto a los hechos presenciados en el entorno virtual. El ser testigo dentro de un acontecimiento concreto, implica al usuario en la toma de conciencia, generando una opinión crítica al respecto y fomentando su participación en el mundo real. La ubicuidad dual, término acuñado por Nonny de la Peña, sería el pilar fundamental de estas obras, cuya obtención se alcanza de la creación de discursos inmersivos con el foco en el efecto presencia. Si bien, ya existía una voluntad de ubicuidad en el cine militante de los años 60, este fenómeno en la actualidad y bajo el influjo de las TIC e Internet se ve amplificado, considerando la RV como uno de los medios capaces de promover la ubicuidad dual. 
En la década de 1960, el cine militante buscaba compartir y difundir estrategias y herramientas para apoyar las luchas políticas del mundo. Jean-Luc Godard lo explicó en 1969 de la siguiente forma: "Una película [militante] es como una alfombra voladora que puede viajar a cualquier lugar. No hay magia. Es trabajo político" (Emmelhainz, 2016: 96).

Esta ubicuidad dual (Peña et al, 2010), como nueva estrategia de activismo audiovisual a través del uso de tecnología de realidad virtual, se centra en crear películas con RV que nos sitúan en el lugar de los hechos, nos pone en la escena, dentro de la diégesis de la no ficción, siendo conscientes de que no estamos allí, pero al mismo tiempo sintiendo la experiencia como si realmente fuésemos testigos, siendo esta ubicuidad o presencia dual lo que nos permite acceder a los sentimientos de empatía de la audiencia, configurando estos metrajes documentales virtuales como herramientas efectivas para la transformación social.

\subsection{VRCinema como generador de conciencia en la audiencia}

Según McRoberts (2018), el cine de RV de no ficción se puede distinguir de otras formas de RV al tratar de implicar a las audiencias con historias reales, donde el sentido de presencia tiene la intención de generar en el público un compromiso empático como agente de transformación social. En la misma línea, Mato Brautović, Romana John y Marko Potrebica (2017) afirman que los autores audiovisuales están dirigiendo la atención hacia el hecho de que el contenido en RV es un buen modo para involucrar a la audiencia con las noticias y promover su empatía. Kate Nash, sugiere que la habilidad de la RV para promover una respuesta en relación al otro (sufrimiento distante) no es imposible, pero tampoco está garantizada por el mero hecho de construir el discurso mediático con esta tecnología, sino que depende de la manera en la que la experiencia virtual es diseñada (Nash, 2018: 121).

Sontag (2003: 34), en relación a la habilidad de crear una respuesta moral en la audiencia por parte del género documental, afirma que, como espectadores, se nos sitúa como voyeurs, independientemente de que queramos o no serlo, aunque la respuesta moral tampoco está garantizada por el relato de no ficción. En cualquier caso, la importancia de la presencia para producir una actitud de testigo de unos hechos concretos, sugiere el valor de la realidad virtual como plataformatestimonio (Nash, 2018: 126). La simulación, en contraposición a la representación, se erige como la fuerza moral que incorpora la RV en el activismo audiovisual.

El trabajo de Dan Archer y Katharina Finger (2018), sobre la generación de empatía en la audiencia de productos audiovisuales con RV, llega a la conclusión de que los formatos de RV dan lugar a respuestas empáticas más altas que los medios tradicionales, resultando en una mayor probabilidad de que estos sujetos tomen parte en las acciones políticas o sociales, después de haber accedido al contenido de la simulación en el entorno virtual. También demuestran, que los usuarios que experimentaron con contenidos RV, afirmaron estar más dispuestos a tomar 
acciones o tratar de descubrir e investigar más sobre los temas expuestos. Desde el plano formal, su investigación confirma que la confianza en el narrador por parte del narratario es esencial para construir empatía y un mayor compromiso en relación a la narración. Esto puede ser posibilitado a través de mantener la figura del narrador visible constantemente.

También son más atractivas aquellas historias con un protagonista claro que guía al usuario a través de la diégesis en RV, y este poder de atracción dota a la pieza de un mayor impacto sobre el espectador. La inmersión y la presencia son fundamentales, pero no son suficientes para contrarrestar la falta del interés del usuario, o si este ya está familiarizado en exceso con el tema sobre el que versa la experiencia cinemática de RV. Es importante indicar que la hipótesis de este estudio parte de los tratamientos con RV a largo plazo para conseguir cambios de comportamiento, desde la perspectiva que estos tendrán un mayor impacto en el individuo, consiguiendo una mayor probabilidad en la obtención de los resultados propuestos. Resulta clave indicar, que estos tratamientos se basaban en proyectar sobre los individuos producciones documentales en video 360, con dispositivo $H M D$, cuyas historias versan sobre el campo de refugiados de Calais, Francia; un día en la vida de una chica de Kenya en la frontera de Tanzania y la colaboración de RYOT y el Sierra Club en Alaska, narrado este último por Jared Leto. Estos contenidos pueden consumirse en sistemas inmersivos y no inmersivos (desktop experience), y en relación a estos concluyen que la inmersión no demostró una diferencia significativa en los resultados, pero sí que el formato inmersivo de RV es más efectivo para producir respuestas empáticas, y que existe una relación directa entre el nivel de inmersión y el deseo del espectador en tomar acciones respecto al conflicto expuesto.

De manera paralela, Donghee Shin llevó a cabo el estudio Empathy and embodied experience in virtual environment: To what extent can virtual reality stimulate empathy and embodied experience? (2018). Los resultados de su investigación implican que los rasgos personales de los usuarios se correlacionan con la inmersión en la realidad virtual: la experiencia del usuario en la realidad virtual depende de los rasgos individuales, lo que a su vez influye en la forma en que los usuarios se sumergen en un entorno de RV, coincidiendo con la tesis de Reinhard y Dervin (2012).

El resultado final de la experiencia del cine de realidad virtual, como generador de conciencia en la audiencia, depende del modo en el que ésta se diseñe, de su desarrollo, desde los elementos documentales, textuales y formales del lenguaje, y desde los elementos de significación del discurso, que en su suma dan lugar a la experiencia del usuario, en una analogía directa con el plano estético del modelo MDA de análisis de videojuegos (Hunicke, LeBlanc y Zubek, 2004), siendo el espectador, su experiencia y bagaje personal y su nivel de instrucción los que en última instancia dan lugar al sentimiento empático en relación a la simulación, lo que en definitiva indica la intervención del plano subjetivo. 


\section{Metodología}

La metodología utilizada en esta investigación, de carácter cualitativo, se basa en una primera revisión y análisis de las fuentes bibliográficas en relación a la hipótesis de trabajo, para establecer un primer acercamiento sobre el impacto de la RV en el ámbito del activismo audiovisual contemporáneo. Una vez estudiadas las principales referencias e investigaciones llevadas a cabo sobre el poder generador de empatía en las audiencias por parte de las producciones VRCinema de no ficción, se aborda el análisis descriptivo de dos de las piezas de RV producidas por Emblematic Group y dirigidas por Nonny de la Peña y por Cassandra Herrman y Lauren Mucciolo, la primera película de RV, Hunger in L.A. (2012), y After Solitary (2017).

Para el estudio de las obras audiovisuales de RV, se ha diseñado un modelo de análisis que parte del triángulo inmersivo: las pantallas, el formato y los espectadores (Cortés-Selva, 2015: 355) y lo actualiza, usando las variables que establecen Archer y Finger (2018) en los resultados de su investigación, así como algunas de las características visuales e interactivas de la inmersión digital establecidas por Eva Domínguez-Martín (2015). El modelo de análisis se compone de tres bloques. En el primer bloque, se revisan los elementos contextuales y documentales, así como las interfaces de producción y consumo; en el segundo apartado, se analizan los elementos textuales y de lenguaje; y en último lugar, se estudian los elementos del discurso y su significación, conectando con la experiencia de la audiencia. Se establecen las relaciones de causa-efecto y las conexiones entre cada uno de los elementos de cada columna, según se muestra en el modelo de análisis (Tabla 1). La visualización de las obras analizadas se ha llevado a cabo con un visor RV Oculus Rift, y el acceso a las obras ha sido principalmente a través de la plataforma SteamVR.

Tabla 1. Modelo de análisis para producciones de VRCinema.

\begin{tabular}{|c|c|c|}
\hline $\begin{array}{lr}\text { 1. Elementos } \\
\text { contextuales y } \\
\text { documentales. } \\
\text { (Interfaces de } \\
\text { producción } \\
\text { consumo) }\end{array}$ & $\begin{array}{l}\text { 2. Elementos textuales y de lenguaje. } \\
\text { (Interfaz cultural) }\end{array}$ & $\begin{array}{l}\text { 3. Elementos de discurso y } \\
\text { significación. (Experiencia de } \\
\text { usuario) }\end{array}$ \\
\hline \multirow{3}{*}{$\begin{array}{l}\text { Documentación y } \\
\text { archivos reales } \\
\text { del suceso que se } \\
\text { simula }\end{array}$} & Audio real de los acontecimientos & \multirow[t]{3}{*}{ Nivel de inmersión } \\
\hline & Producción sonora (foley) & \\
\hline & $\begin{array}{l}\text { Imagen de registro en video } 360 / \\
\text { gráficos 3D }\end{array}$ & \\
\hline
\end{tabular}




\begin{tabular}{|c|c|c|c|c|c|}
\hline \multirow[t]{5}{*}{$\begin{array}{l}\text { 1. Elementos } \\
\text { contextuales y } \\
\text { documentales. } \\
\text { (Interfaces de } \\
\text { producción y } \\
\text { consumo) }\end{array}$} & \multicolumn{3}{|c|}{$\begin{array}{l}\text { 2. Elementos textuales y de lenguaje. } \\
\text { (Interfaz cultural) }\end{array}$} & \multicolumn{2}{|c|}{$\begin{array}{l}\text { 3. Elementos de discurso y } \\
\text { significación. (Experiencia de } \\
\text { usuario) }\end{array}$} \\
\hline & \multicolumn{3}{|c|}{ Visualización de datos 3D multicapa } & & \\
\hline & \multicolumn{3}{|c|}{ Captura volumétrica 3D } & & \\
\hline & \multicolumn{3}{|c|}{ Fotogrametría } & & \\
\hline & \multicolumn{3}{|l|}{ Hologramas } & & \\
\hline \multirow[t]{6}{*}{ Temática } & & & & Social & \multirow[t]{6}{*}{ Nivel de disfrute } \\
\hline & & & & $\begin{array}{l}\text { Medio } \\
\text { Ambiente }\end{array}$ & \\
\hline & & & & Política & \\
\hline & & & & Género & \\
\hline & & & & Identidad & \\
\hline & & & & $\begin{array}{l}\text { Conflictos } \\
\text { armados }\end{array}$ & \\
\hline \multirow{5}{*}{$\begin{array}{lr}\text { Interfaces } & \text { de } \\
\text { producción } & \text { y } \\
\text { estilo gráfico } & \end{array}$} & $\begin{array}{l}\text { Cámaras } \\
360\end{array}$ & Registro video & gráfico 360 & \multirow{5}{*}{\multicolumn{2}{|c|}{ Realismo perceptual }} \\
\hline & $\begin{array}{l}\text { Software } \\
\text { fotogrametrí } \\
\text { a }\end{array}$ & Fotogrametría & & & \\
\hline & Software 3D & $\begin{array}{l}\text { Gráficos 3D } \\
\text { hiperrealistas }\end{array}$ & $\begin{array}{l}\text { Gráficos } \\
3 \mathrm{D} \quad \mathrm{NO} \\
\text { realistas }\end{array}$ & & \\
\hline & $\begin{array}{l}\text { Software } \\
\text { holografía }\end{array}$ & \multicolumn{2}{|l|}{ Hologramas } & & \\
\hline & Escáner 3D & \multicolumn{2}{|c|}{ Gráficos 3D hiperrealistas } & & \\
\hline
\end{tabular}




\begin{tabular}{|c|c|c|c|c|c|}
\hline \multirow[t]{2}{*}{$\begin{array}{l}\text { 1. Elementos } \\
\text { contextuales y } \\
\text { documentales. } \\
\text { (Interfaces de } \\
\text { producción y } \\
\text { consumo) }\end{array}$} & \multicolumn{3}{|c|}{$\begin{array}{l}\text { 2. Elementos textuales y de lenguaje. } \\
\text { (Interfaz cultural) }\end{array}$} & \multicolumn{2}{|c|}{$\begin{array}{l}\text { 3. Elementos de discurso y } \\
\text { significación. (Experiencia de } \\
\text { usuario) }\end{array}$} \\
\hline & $\begin{array}{l}\text { Motor de } \\
\text { Videojuegos }\end{array}$ & Unity / Unreal & & & \\
\hline \multirow[t]{2}{*}{$\begin{array}{l}\text { Interfaces de } \\
\text { consumo }\end{array}$} & \multicolumn{3}{|l|}{ Desktop } & \multirow[t]{2}{*}{$\begin{array}{l}\text { Impacto } \\
\text { emocional }\end{array}$} & \multirow{2}{*}{$\begin{array}{l}\text { Nivel de } \\
\text { comodidad }\end{array}$} \\
\hline & \multicolumn{3}{|c|}{$\begin{array}{l}\text { HMD (visor gafas RV) (Oculus Rift, HTC } \\
\text { Vive, etc..) }\end{array}$} & & \\
\hline \multirow{2}{*}{$\begin{array}{l}\text { Distribución } \\
\text { Distribución }\end{array}$} & Festivales & \multicolumn{2}{|c|}{ Consumo colectivo } & \multirow{2}{*}{\multicolumn{2}{|c|}{$\begin{array}{l}\text { Nivel de comodidad } \\
\text { Nivel de comodidad }\end{array}$}} \\
\hline & $\begin{array}{l}\text { Plataformas } \\
\text { online }\end{array}$ & \multicolumn{2}{|c|}{ Consumo individual } & & \\
\hline \multirow[t]{3}{*}{$\begin{array}{l}\text { Punto de vista del } \\
\text { espectador } \\
\text { (Ocularización) }\end{array}$} & \multirow[t]{2}{*}{$1^{\text {a }}$ Persona } & $\begin{array}{l}\text { Corporeidad } \\
\text { virtual SI / } \\
\text { NO }\end{array}$ & \multirow{2}{*}{$\begin{array}{l}\text { Focalizaci } \\
\text { ón interna } \\
\text { y variable } \\
\text { Estilo } \\
\text { directo } \\
\text { (diálogos) }\end{array}$} & \multirow{3}{*}{\multicolumn{2}{|c|}{ Confianza en el narrado }} \\
\hline & & $\begin{array}{l}\text { Ocularizació } \\
\mathrm{n} \quad \text { interna } \\
\text { primaria }\end{array}$ & & & \\
\hline & $3^{\mathrm{a}}$ Persona & $\begin{array}{l}\text { Ocularizació } \\
\text { n cero o } \\
\text { espectatorial } \\
\text { (Testigo) }\end{array}$ & $\begin{array}{l}\text { Focalizaci } \\
\text { ón externa } \\
\text { o cero } \\
\text { (estilo } \\
\text { indirecto) }\end{array}$ & & \\
\hline \multirow[t]{2}{*}{ Estructura } & $\begin{array}{l}\text { Con hilo } \\
\text { narrativo }\end{array}$ & Ramificada & Lineal & \multirow{2}{*}{\multicolumn{2}{|c|}{ Realismo perceptual }} \\
\hline & Continua & $\begin{array}{l}\text { Fragmentad } \\
\text { a }\end{array}$ & Arbitraria & & \\
\hline $\begin{array}{l}\text { Navegación e } \\
\text { interactividad }\end{array}$ & $\begin{array}{l}\text { Movimiento } \\
\text { libre del } \\
\text { espectador } \\
\text { SI/NO }\end{array}$ & $\begin{array}{l}\text { Movimiento } \\
\text { a través de } \\
\text { menús }\end{array}$ & $\begin{array}{l}\text { Acciones } \\
\text { O } \\
\text { actividade } \\
\text { s }\end{array}$ & Nivel de in & ersión \\
\hline
\end{tabular}




\begin{tabular}{|c|c|c|c|c|}
\hline $\begin{array}{l}\text { 1. Elementos } \\
\text { contextuales y } \\
\text { documentales. } \\
\text { (Interfaces de } \\
\text { producción y } \\
\text { consumo) }\end{array}$ & \multicolumn{3}{|c|}{$\begin{array}{l}\text { 2. Elementos textuales y de lenguaje. } \\
\text { (Interfaz cultural) }\end{array}$} & $\begin{array}{l}\text { 3. Elementos de discurso y } \\
\text { significación. (Experiencia de } \\
\text { usuario) }\end{array}$ \\
\hline $\begin{array}{l}\text { Sistemas de } \\
\text { interacción, } \\
\text { mecánicas de la } \\
\text { experiencia }\end{array}$ & Narrativas & $\begin{array}{l}\text { Seleccionar } \\
\text { Coger } \\
\text { Rotar } \\
\text { Examinar } \\
\text { Abrir } \\
\text { Explorar }\end{array}$ & $\begin{array}{l}\text { Escribir } \\
\text { Disparar } \\
\text { Atacar } \\
\text { Arrojar } \\
\text { Aplicar }\end{array}$ & $\begin{array}{l}\text { Nivel de inmersión } \\
\text { Impacto emocional }\end{array}$ \\
\hline $\begin{array}{l}\text { Acción en la } \\
\text { trama }\end{array}$ & $\begin{array}{l}\text { Espectador } \\
\text { incide y } \\
\text { actúa }\end{array}$ & $\begin{array}{l}\text { Espectador - } \\
\text { Testigo, no } \\
\text { influye ni } \\
\text { actua en la } \\
\text { trama }\end{array}$ & & $\begin{array}{l}\text { Nivel de inmersión } \\
\text { Impacto emocional }\end{array}$ \\
\hline $\begin{array}{l}\text { Movimientos de } \\
\text { cámara }\end{array}$ & $\begin{array}{l}\text { Controlados } \\
\text { por el } \\
\text { usuario }\end{array}$ & $\begin{array}{l}\text { Impuestos } \\
\text { por la } \\
\text { diégesis }\end{array}$ & & $\begin{array}{l}\text { Nivel de inmersión } \\
\text { Impacto emocional }\end{array}$ \\
\hline Puntos de vista & $\begin{array}{l}\text { De un solo } \\
\text { personaje }\end{array}$ & $\begin{array}{l}\text { De más de } \\
\text { un personaje }\end{array}$ & $\begin{array}{l}\begin{array}{l}\text { Relato } \\
\text { multiform } \\
\text { e }\end{array} \\
\begin{array}{l}\text { Relato } \\
\text { puzle }\end{array}\end{array}$ & $\begin{array}{l}\text { Nivel de inmersión } \\
\text { Impacto emocional }\end{array}$ \\
\hline
\end{tabular}

Fuente: Elaboración propia.

\section{Resultados}

A continuación, se exponen los resultados del análisis de las obras objeto de estudio, abordando las características visuales e interactivas, así como los aspectos recogidos en el modelo de análisis. La producción de Emblematic Group versa sobre conflictos sociales, con un marcado objetivo en relación a crear conciencia y reflexión crítica en el espectador, sobre los acontecimientos e historias que componen estos relatos audiovisuales virtuales.

\subsection{Hunger in L.A. (2012)}

La primera de las producciones llevadas a cabo por Emblematic Group en colaboración con la USC School of Cinematic Arts, escrita, dirigida y producida por Nonny de la Peña, nace de unas grabaciones que recogió una colaboradora de ella 
mientras hacía sus prácticas, Michaela Kobsa-Mark. Fue la primera película de RV presentada en un festival de cine, en Sundance 2012. Esta obra parte del registro fotográfico y de audio sobre la crisis de los bancos de alimentos en Los Ángeles (CA), mientras la autora llevaba a cabo una investigación sobre la situación abrumadora y las limitaciones con las que contaban, debido al incremento de la hambruna entre la población. La producción fue llevada a cabo sin presupuesto, pues en este momento no contaban con ningún tipo de financiación o ayuda para producirla, ya que la RV todavía se encontraba en un estado incipiente. La producción sonora, a partir de los audios reales del suceso que trata, otorga a la obra el potencial de sumergir al espectador dentro de la fila de gente que está esperando para recibir alimentos, a las puertas de una parroquia. Los modelos 3D fueron donados y modelados por voluntarios, siendo todo el entorno una reconstrucción sintética 3D del lugar y los acontecimientos. El nivel de realismo alcanza cotas suficientes para servir como medio de inmersión, el tratamiento gráfico pretende ser lo más preciso posible, con los medios con los que se contaba en ese momento. Los elementos textuales aparecen al principio de la obra, para contextualizar la situación extrema del hambre en niños y adultos en la ciudad californiana, y el sonido diegético apoya el relato visual a través de situar a la audiencia haciendo la fila por alimentos. De temática social, expone y denuncia la alarma que suscita el hambre en sociedades avanzadas, desigualdad en relación al acceso para cubrir las necesidades básicas de cualquier ser humano.

Hunger is based on real audio of an incident at a food bank outside the First Unitarian Church in downtown Los Angeles: a diabetic man waiting on line collapsed when his blood sugar level dropped too low, and an ambulance had to be called. Emblematic Founder Nonny de la Peña was inspired to recreate the event in VR, using animated figures to represent the participants (Emblematic Group, 2019).

El visor utilizado, para su visionado en Sundance, es la primera versión de lo que más tarde serían las gáfas de RV Oculus Rift, y fue construido en la USC por Mark Bolas, Palmer Luckey, Thai Phan y Evan Suma. Esta obra pionera en su género, utiliza Unity como motor de videojuegos, y fue desarrollada en 3D para un primer prototipo de los visores de RV actuales. Aunque la pieza puede verse en Youtube, esta versión no es interactiva, ni en 360, por lo que para poder visualizarla en HMD está disponible en la plataforma de SteamVR, para Oculus y HTC Vive, donde fue publicada el 2 de febrero de 2017, cinco años después de su estreno en Sundance.

A pesar del momento de su creación, del estado germinal de las técnicas y métodos para producir películas de RV, esta obra presenta un alto nivel de inmersión y un realismo perceptual que toca la emoción de la audiencia en relación al tema que nos presenta (Figura 1). El impacto emocional que se consigue es el resultado de la combinación del audio real de los eventos con los gráficos 3D del entorno virtual. Observamos un nivel de ocularización cero, y una focalización interna fija, "los acontecimientos se filtran a través de la conciencia de un único personaje, como centro de la perspectiva" (Jost y Gaudreault, 2010: 139), en este caso el espectador 
ocupa el lugar (y punto de vista) de una de las personas que esperan en la fila. La confianza en el narrador en este caso no existe, siendo una experiencia presencial que trata de ubicar al espectador en la piel de una de esas personas que conforman el suceso. No existe tampoco corporeidad, por tanto, estos dos componentes generadores de empatía no participan de la experiencia estética del usuario.

Fuente: Youtube. https://bit.ly/2RYulKM

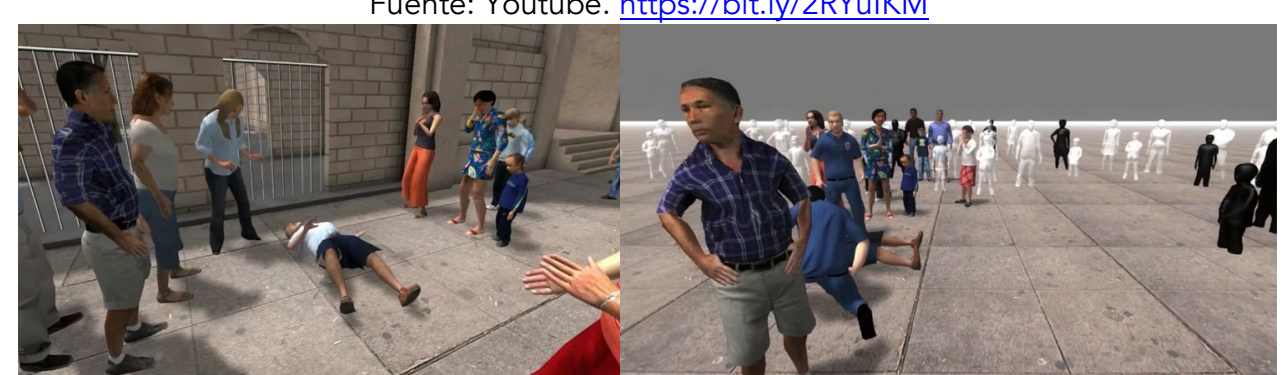

Figura 1. Fotogramas de dos instantes de Hunger in L.A., el momento de la crisis diabética (izq.) y en el segundo acto, donde se plantea la reflexión de un modo más lírico (dcha.) Emblematic Group y USC School of Cinematic Arts (C).

La estructura narrativa de esta obra de aproximadamente tres minutos de duración es lineal, y se fragmenta en dos apartados, el primero que nos relata el suceso, y el segundo, que nos plantea una reflexión acerca de esta problemática social. La navegación es visual, a través del entorno virtual, lo que facilita la generación del efecto inmersivo en la diégesis de la película. El usuario no puede interactuar, no participa activamente en la acción de la trama, los movimientos de cámara son controlados por el usuario, desde un único punto de vista en primera persona. En definitiva, aunque los gráficos no son completamente realistas, el dispositivo VR rastrea los pasos y movimientos, y combinados con audio real, proporcionan una experiencia evocadora que provoca respuestas emocionales profundas de los usuarios.

\subsection{After Solitary (2017)}

Esta pieza de VRCinema producida por Emblematic Group y Frontline PBS, ha sido desarrollada a partir del motor de videojuegos Unity. Ganadora del gran premio del jurado en el SXSW Film Festival 2017 y del máximo galardón del World VR Forum, ha sido dirigida por Cassandra Herrman y Lauren Mucciolo, y presenta una evolución técnica a través de tecnología de captura de video volumétrico y fotogrametría sobre fotografía 360, fácil de observar si la comparamos con las primeras producciones de Emblematic Group como Hunger in L.A. (2012). Cuenta la historia de Kenny Moore, un ex convicto de 39 años, recién liberado de prisión donde pasó años en una celda de aislamiento. La obra parte de una rigurosa documentación previa, de archivos de audio reales registrados en la prisión de Maine y usados de manera diegética, insertados sobre el sonido ambiente en relación a lo que el protagonista narra, además de una banda sonora, a modo de música de fondo (sonido extradiegético), para aumentar la sensación dramática del relato. La pista principal de audio está compuesta por la voz de Kenny, mientras él 
de manera presencial nos cuenta su experiencia personal (sonido diegético). Este uso de elementos textuales que parten de la documentación de archivo, sonido real, registro fotográfico 360 de las localizaciones reales, fotogrametría de estos espacios y captura volumétrica 3D del personaje principal, dotan a la obra de un alto nivel de inmersión, dentro del discurso de la obra y su significación.

De temática social, profundiza en la situación de los presos de los centros penitenciarios y la violencia y prácticas extremas a los que son sometidos. En esta obra concreta, se denuncian las prácticas de aislamiento en la prisión de Maine, y contrasta los datos experienciales del protagonista con estudios, que confirman que este tipo de procedimientos son contraproducentes para la reinserción de los convictos, y aumentan la probabilidad de su retorno una vez han cumplido su condena. Refuerza el carácter de documento que nos acerca a una realidad para que, en primer lugar, la sociedad norteamericana sea conocedora del conflicto existente y su problemática, en relación al sistema judicial y penitenciario en funcionamiento. Presenta un alto nivel de realismo perceptual, como testigos, se nos ubica en el registro 360 de unos espacios que podemos navegar a través de nuestra mirada, y el personaje principal y único aparece ante nosotros en un registro de captura volumétrica 3D de alto realismo, para el que han sido utilizadas cámaras 360 y cámaras DSLR, sincronizadas con sensores de captura 3D, con los que se ha tomado el registro de Kenny, que posteriormente ha sido ubicado junto al espectador en los espacios virtuales 360 a través de fotogrametría.

El metraje de After Solitary (2017) es una ventana visceral a la práctica del aislamiento, que la prisión estatal de Maine comenzó a reducir mientras Moore estaba encerrado. La prisión también comenzó a ofrecer clases de rehabilitación a los reclusos y desde 2011, las tasas de violencia y autolesión han disminuido drásticamente. Pero los estudios muestran que los reclusos, que han pasado mucho tiempo en aislamiento, tienen más probabilidades de ser enviados de regreso a prisión (Taddonio, 2017).

Esta película de RV fue presentada en el SXSW Film Festival 2017, Milano Film Festival 2018 y en el World VR Forum 2018. Además, fue publicada en su versión para desktop en las plataformas Youtube (18 de abril de 2017) y Facebook, y en SteamVR (18 de marzo de 2019) para su distribución gratuita para dispositivos HMD. Esta estrategia, de mantener ambos formatos, amplía el acceso a la obra por parte de la audiencia, debido a la todavía lenta introducción de los visores RV como tecnología de consumo habitual. El impacto emocional es significativo en la versión para visor RV, y el nivel de comodidad es óptimo en ambos casos, teniendo también distribución tanto a nivel de festivales (consumo colectivo), como a nivel de plataformas.

En cuanto al punto de vista, el espectador es situado en primera persona, como testigo que acompaña al personaje principal, sin corporeidad, ya que no se nos ofrece una representación virtual de nuestro cuerpo en el entorno de la obra. Según 
Todorov (1966), estaríamos ante un narrador-personaje. Desde la focalización de Genette (1972), identificamos una focalización interna fija: "cuando el relato da a conocer los acontecimientos como si estuviesen filtrados por la consciencia de un solo personaje" (Jost y Gaudreault, 2010: 139). Observamos una ocularización cero o "nobody's shot" (Jost y Gaudreault, 2010: 143). El resultado es un alto nivel de empatía con el narrador-personaje, a partir de la confianza que genera la presencia y conexión del narrador, siendo este, al mismo tiempo, el actor principal de la pieza.

Fuente: Youtube. https://bit.ly/31dallS

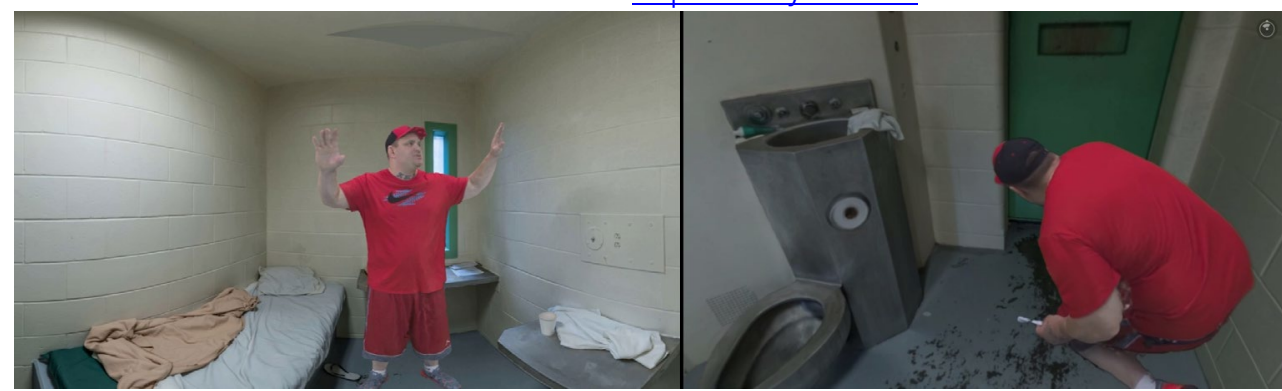

Figura 2. Fotogramas de dos instantes del segundo bloque de After Solitary. Emblematic Group y Frontline PBS @

La estructura de esta obra audiovisual RV de 9 minutos de duración, con hilo narrativo de la historia cronológica de Kenny durante su reclusión, se fragmenta linealmente en cuatro bloques. En la primera parte, el espectador es ubicado en la habitación de Kenny, donde se presenta a sí mismo en voz en off mientras se encuentra mirando por la ventana (Figura 3). Tras un fundido a negro comienza la segunda parte que se subdivide en cuatro apartados, en la celda de aislamiento (Figura 2). En el primer acto, el personaje principal está de pie, mientras nos habla, terminando con la sobreimpresión de texto sobre la pared una vez Kenny ha desaparecido a través de un fade out. El segundo bloque comienza con la aparición fade in de Kenny sentado en la cama, mientras continúa relatando la dureza de estar confinado en una celda de aislamiento. Más tarde, vuelve a aparecer arrodillado delante de la puerta de la celda (tercer subapartado), mientras nos explica los métodos para poder estar en contacto con otros presos a través de la pequeña rendija inferior de la entrada del cubículo. Vuelve a desaparecer de la imagen y se nos vuelve a presentar de pie (cuarto subapartado), donde termina el relato en la celda. Con un fundido a negro pasamos al tercer bloque de la narración, que se divide en dos apartados. En el primero, Kenny está detrás de la ventana de la celda, se nos sitúa en el pasillo, y se nos muestra un video sobre la violencia de los oficiales de prisiones en los procedimientos de extracción de los reclusos (Figura 3), continúa la voz en off de Kenny, y tras un fundido a negro volvemos a la celda, donde comienza a entrar luz y aparecen unas fotografías de la familia de Kenny, mientras continuamos escuchando al narrador-personaje. Tras un fundido a negro, pasamos al último bloque donde volvemos a la habitación. Se alterna la imagen del interlocutor sentado en la cama con textos flotantes, que muestran datos sobre estudios recientes, que abordan la situación extrema de los presos aislados. El tipo 
de estructura narrativa empleada apoya el discurso, aportándole realismo a los hechos relatados.

Fuente: Youtube. https://bit.ly/36Fbhfz

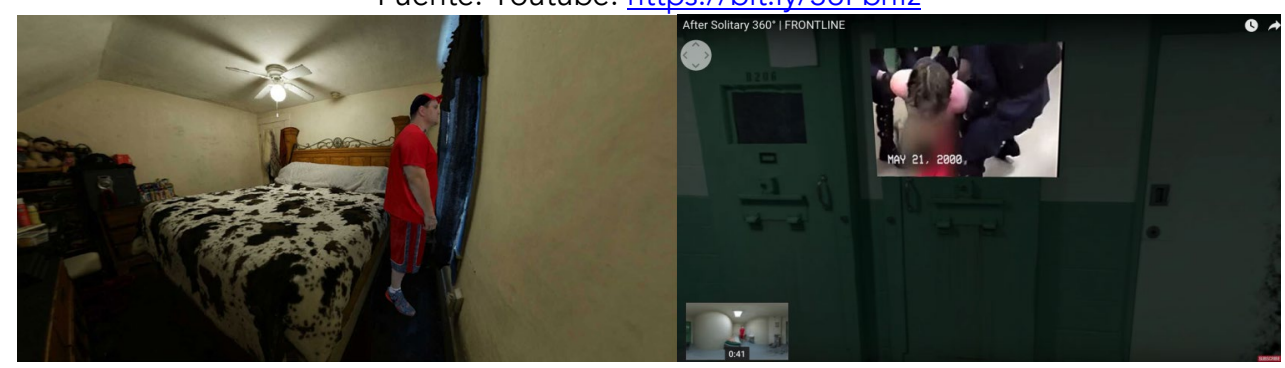

Figura 3. Fotogramas de dos instantes de After Solitary, el inicio (izq.) y la primera parte del tercer acto, justo antes de que se muestre el video sobreimpreso en el entorno virtual (dcha.). Emblematic Group y Frontline PBS @ .

En relación a la navegación e interactividad, esta es inexistente, al menos en las versiones de la pieza para desktop y para visores RV desde la plataforma SteamVR, lo que reduce en cierto modo el nivel de inmersión, aunque no de manera drástica, pues la pieza persigue situarnos como testigos de la situación que el relato transmite, por lo tanto, tampoco existen mecánicas, más allá de la navegación de nuestra mirada por el entorno, donde se nos van mostrando diferentes materiales fotográficos, de video y textuales, con información periodística que respalda el discurso.

Los movimientos de la cámara son prácticamente inexistentes. Se observan cambios del punto de vista desde donde vemos la acción que acontece, a través de los fundidos a negro. El cambio de posición del personaje principal en el espacio, es introducido a través de efectos fade in y out del mismo, que desaparece para reaparecer en una ubicación distinta. Estas alteraciones vienen impuestas por la trama, según la estructura narrativa creada, con el fin de generar un impacto emocional en el espectador, potenciado por el uso de un solo personaje, en una suerte de relato puzle.

La obra nos sumerge en una realidad física y emocional difícilmente accesible en nuestra vida real, para hacernos reflexionar sobre la efectividad de los métodos que se emplean, para la reinserción de los convictos, en las cárceles estadounidenses. Esta película de realidad virtual se complementa con el documental Last Days of Solitary (Edge y Mucciolo, 2017), dentro de la serie documental producida por Frontline.

\section{Discusión y conclusiones}

Tras el análisis de las obras objeto de estudio, a partir del modelo empleado (Tabla 1), y atendiendo a los factores incluidos en la columna 3 (Experiencia de usuario), observamos en Hunger in L.A y en After Solitary, el uso común de los siguientes 
recursos de la columna 2, elementos textuales y de lenguaje, clasificados por las categorías de la columna 1 (Interfaces de producción y consumo). Con el fin de alcanzar un nivel de inmersión, en ambas producciones detectamos registro de audio real de los acontecimientos y producción sonora, que demuestran un trabajo de documentación e investigación previa sobre los temas tratados. Siguiendo con el nivel de inmersión e impacto emocional en el marco de la experiencia de usuario, ambas obras presentan como sistema de navegación el movimiento libre del espectador, pero no incluyen ningún otro tipo de acción por parte del mismo, por lo que en relación a los sistemas de interacción y mecánicas no se observa ningún recurso, más allá de la narrativa, que guía la experiencia de la audiencia. En relación a la acción en la trama, identificamos la estrategia de espectador-testigo, sin capacidad de influir en esta. Dentro de este mismo factor de la experiencia de usuario, la posición de la cámara es en ambos casos impuesta por la diégesis de la obra, y a su vez, los movimientos de esta son controlados por el usuario desde su capacidad de orientar su mirada en el entorno virtual. Por último, los puntos de vista empleados atienden a un único personaje-testigo.

En relación al nivel de disfrute, conectado este factor con la temática como elemento contextual y documental, se observa una clara orientación social y política en ambas obras. Así mismo, en la construcción de realismo perceptual, identificamos primero el uso de interfaces de producción comunes como los motores de videojuegos empleados para la creación de ambas producciones. Con el fin de crear realismo perceptual, la estructura de ambos metrajes es lineal y fragmentada, con hilo narrativo. En cuanto a generar impacto emocional, destacamos el uso de visores HMD, sistemas de interacción basados únicamente en la cualidad narrativa, sin mecánicas ni acciones posibles por parte de la audiencia. Según el nivel de comodidad, se observa la distribución de ambas obras en festivales y plataformas online, facilitando un consumo individual y colectivo, a través, tanto de visores HMD como de reproductores convencionales desktop.

Hunger in L.A, difiere de After Solitary, principalmente en el empleo de recursos y estrategias, tales como imagen sintética de gráficos $3 D$, en la primera, mientras que, en la segunda, el método de producción utiliza registro de video 360, captura volumétrica 3D y fotogrametría. Del mismo modo, y en relación a la creación de realismo perceptual, la primera obra emplea interfaces de producción tales como software 3D, gráficos con intención realista, mientras que After Solitary se construye con los registros mencionados previamente a través de cámaras 360, cámaras DSLR sincronizadas con sensores de captura 3D de movimiento. También presentan diferentes estrategias en cuanto a la creación de confianza en el narrador. En Hunger in L.A no existe tal confianza, pues el espectador es uno más en el acontecimiento, presentando un nivel de ocularización cero y una focalización interna fija. Sin embargo, en After Solitary identificamos la presencia del narrador en el personaje principal, con un nivel de ocularización cero, el espectador es testigo de los acontecimientos. 
El cine de RV se afianza dentro de los medios de producción y consumo de la industria, a pesar de las reticencias existentes en sus primeros años de irrupción. Cada día más estandarizada, no solo para dispositivos HMD, sino también dentro de las corrientes transmediáticas que aprovechan las posibilidades del video 360 para pantalla. El videoactivismo de realidad virtual se confirma como una de las corrientes que han fomentado el desarrollo y evolución de las técnicas y métodos de producción del VRCinema. Su impacto sobre la audiencia, capaz de generar conductas en los espectadores, lo convierte en una potente herramienta de acción social, cuyo poder educativo y prosocial reside en la ubicuidad dual, una de sus principales características.

La ubicuidad dual es producto del efecto inmersión producido por el realismo perceptual, que a su vez es fruto del registro de sonido real y de la reconstrucción visual hiperrealista. El nivel de comodidad del usuario y la creación de confianza en el narrador también participan en el impacto emocional que genera el efecto de estar en otro lugar, siendo conscientes de ello, encontrándonos en dos espacios en un mismo tiempo.

Se observa una evolución técnica en la imagen en movimiento virtual, al comparar la obra inicial de 2012 con una de las producciones de 2017. Esta mejora incide en un aumento del nivel de inmersión y, por ende, del nivel de impacto susceptible para transmitir valores y educar en conductas. A pesar de que las obras analizadas no presentan sistemas de interacción directos, ni mecánicas dentro de la experiencia, y los sistemas de navegación únicamente ofrecen al espectador el movimiento libre por el entorno virtual, son suficientes en el proceso de generación de impacto emocional en la audiencia.

Aunque ya se ha indicado la dificultad de garantizar la impresión de emoción en el usuario por parte de los discursos audiovisuales RV de no ficción, dado que el espectador aporta el plano subjetivo de su experiencia en la última fase del hecho comunicativo, se sugiere un potencial que radica en las características indicadas y en la ubicuidad dual como principal agente generador de empatía en la audiencia, por lo que el cine de realidad virtual puede configurarse como un excelente vehículo de acción social.

Las principales características y recursos para dotar a la experiencia audiovisual de $\mathrm{RV}$ de poder educativo, en la generación de conductas prosociales en la audiencia, son el efecto presencia, generado a través de la ilusión de lugar, la supresión de incredulidad y la corporalidad virtual, siendo esta última la única no presente en las obras objeto de estudio, al menos desde la perspectiva de representación del cuerpo tridimensional del espectador en el entorno virtual, factor que no minora la sensación de estar presente en otra realidad espacio temporal. Para ello, se identifica el nivel de ocularización cero y la focalización interna fija como estrategias comunes en las obras analizadas. 
La distribución tanto en plataformas online desktop como para visores HMD induce a pensar en una estrategia múltiple, frente a la lenta inmersión de los visores de realidad virtual como dispositivos de consumo estandarizados. Además, su presencia en festivales de cine es cada vez más significativa, atrayendo a la audiencia a estos nuevos productos audiovisuales.

En las obras analizadas, identificamos una combinación entre reconstrucción de eventos y situaciones reales, con momentos más líricos y poéticos, que sirve para generar el equilibrio de la simulación desde la veracidad de lo real, hasta alcanzar los sentimientos de los espectadores a través de reflexiones visuales y sonoras, además del uso de texto como método común para transmitir datos e información contrastada, con el objetivo de generar una opinión crítica.

Las temáticas se centran en conflictos sociales y de urgencia en la mayoría de los casos, donde el metraje de RV se constituye como vehículo de acción social, transportándonos a lugares físicos y emocionales a los que no tendríamos acceso en nuestra vida diaria, para promover la reflexión sobre estos problemas actuales, que demandan un profundo debate y participación ciudadana. El VRCinema, como el cine en periodos anteriores, sirve como herramienta política y militante. Sus características potencian la conexión de su relato con la audiencia, en un amplio abanico de posibilidades comunicativas todavía por explotar. 


\section{Referencias bibliográficas}

ARCHER, D. y FINGER, K. (2018): Walking in Another's Virtual Shoes: Do 360Degree Video News Stories Generate Empathy in Viewers? Tow Center for Digital Journalism, Columbia University

BARROSO, J. A. G., LAZO, C. M. y COVACHO, E. H. (2012): Comunicación, ubicuidad y aprendizajes. Trabajo presentado en el IV Congreso Internacional Latina de Comunicación Social, Universidad de La Laguna, Tenerife.

BAZIN, A. (1966): ¿Qué es el cine? Madrid: Rialp.

BRAUTOVIĆ, M., JOHN, R., y POTREBICA, M. (2017): Immersiveness of news: how croatian students experienced 360-video news. En International conference on augmented reality, virtual reality and computer graphics (pp. 263-269). Springer, Cham.

CORTÉS-SELVA, L. (2015): Viaje al centro de la inmersión cinematográfica, del cine primitivo al VRCinema. Revista de Ciencias Humanas y Sociales, 31 (4), pp. 352-371.

DOMÍNGUEZ-MARTÍN, E. (2015): Periodismo inmersivo o cómo la realidad virtual y el videojuego influyen en la interfaz e interactividad del relato de actualidad. El profesional de la información, 24 (4), pp. 413-423.

EDGE, D. y MUCCIOLO, L. (productores) (2017): Last Days of Solitary [serie documental]. USA: Frontline.

EMBLEMATIC GROUP (2019): Disponible en: https://emblematicgroup.com/about/

EMMELHAINZ, I. (2016): Cine militante: del internacionalismo a la política sensible neoliberal. Secuencias: revista de historia del cine.

GALÁN, M. (2012a): Cine militante y videoactivismo: los discursos audiovisuales de los movimientos sociales. En I Congreso Internacional de la Red Iberoamericana de Narrativas Audiovisuales (Red INAV). Málaga-Sevilla, 23-25 de mayo de 2012. Editores: Virginia Guarinos, María Jesús Ruiz (pp. 1122-1133). Sevilla: Universidad de Sevilla.

(2012b): El fotograma disidente: del cine militante al videoactivismo. Cambios históricos, políticos y culturales en el cine y la televisión.

GARCÍA-MERÁS, L. (2007): El cine de la disidencia. La producción militante antifranquista (1967-1981). Desencuentros, no. 4, pp. 16-41.

GENETTE, G. (1972): Discurso del relato. Ensayo de método. Figuras III. 
HUNICKE, R., LEBLANC, M., y ZUBEK, R. (2004): MDA: A formal approach to game design and game research. En Proceedings of the AAAI Workshop on Challenges in Game Al, 4 (1), pp. 1722.

ISLAS-CARMONA, O. (2008): El prosumidor. El actor comunicativo de la sociedad de la ubicuidad. Palabra clave, 11 (1), pp. 2.

JOST, F., y GAUDREAULT, A. (2010): El relato cinematográfico: cine y narratología. Barcelona: Ediciones Paidós Ibérica.

MCROBERTS, J. (2018): Are we there yet? Media content and sense of presence in non-fiction virtual reality. Studies in Documentary Film, 12 (2), pp. 101-118.

MESTMAN, M. (2001): La exhibición del cine militante. Teoría y práctica en el Grupo Cine Liberación. En Sel, S. (Compilador), La comunicación mediatizada: hegemonías, alternatividades y soberanías. Buenos Aires: Clacso.

NASH, K. (2018). Virtual reality witness: exploring the ethics of mediated presence. Studies in Documentary Film, 12 (2), pp. 119-131.

PEÑA, N., WEIL, P., LLOBERA, J., GIANNOPOULOS, E., POMÉS, A., SPANLANG, B. y SLATER, M. (2010): Immersive journalism: immersive virtual reality for the firstperson experience of news. Presence: Teleoperators and virtual environments, 19 (4), pp. 291-301.

REINHARD, C. D. y DERVIN, B. (2012): Comparing situated sense-making processes in virtual worlds: Application of Dervin's Sense-Making Methodology to media reception situations. Convergence, 18 (1), pp. 27-48.

SHIN, D. (2018): Empathy and embodied experience in virtual environment: To what extent can virtual, reality stimulate empathy and embodied experience? Computers in Human Behavior, v. 78, pp. 64-73.

SOLANAS, F. E. y GETINO, O. (1973): Cine, cultura y descolonización. Buenos Aires: Siglo Veintiuno.

SONTAG, S. (2003): Devant la douleur des autres. C. Bourgois.

TADDONIO, P. (2017): Last Days of Solitary. After Solitary. Frontline. Disponible en: https://to.pbs.org/2RE21E5

TODOROV, T. (1966): Las categorías del relato. AA. VV. (1974): Análisis estructural del relato. Buenos Aires: Tiempo Contemporáneo. 